\title{
Finite Element Analysis Study on Lattice Structure Fabricated Using Corn Husk Fibre Reinforced Recycled Polystyrene Composite.
}

\author{
Mohammed Shariff Mohamed Sulaiman ${ }^{1}$, Seong Chun Koay $^{1,}{ }^{*}$, Ming Yeng Chan $^{2}$, Hui \\ Leng Choo ${ }^{1}$, Ming Meng Pang ${ }^{1}$ and Thai Kiat Ong $^{2}$ \\ ${ }^{1}$ School of Computer Science and Engineering, Faculty of Innovation \&Technology, Taylor's \\ University, No. 1, Jalan Taylor's, 47500 Subang Jaya, Selangor, Malaysia \\ ${ }^{2}$ Faculty of Engineering and Technology, Tunku Abdul Rahman College University, 53300 Kuala \\ Lumpur, Malaysia.
}

\begin{abstract}
This research investigated the lattice structure fabricated using corn husk fibre reinforced recycled polystyrene composite using Finite Element Analysis (FEA). The material's properties of this composite material were obtained from previous study. Then, the lattice structure of lattice structure was created using $\mathrm{Creo}^{\circledR}$ software and the FEA simulation was done by ANSYS software. In this study, the lattice structures were created using triangular prism and hexagonal prism. The analysis was divided into two conditions: 1) lattice structure with different prism shape and similar surface area, 2) lattice structure with varies of strut thickness and 3) lattice structure with different prism shape and similar lattice parameter. The results show the lattice structure with triangular prism have more structural integrity than hexagonal prism. Then, lattice structure with triangular prism can be built with lesser material but stronger and stiffer than lattice structure with hexagonal prism.
\end{abstract}

\section{Introduction}

In this modern era, natural fibre-reinforced polymer composite (NFRPC) is widely used in many applications such as in automobiles, civil construction and aircraft [1-2]. NFRPC is generally a material that is combination of natural fibres and polymer matrix either to thermoplastic or thermoset and they can be produced via conventional polymer manufacturing process such like injection molding and compression molding [3-4]. Nowadays, NFRPC can turn into 3D printing filament for fuse deposition modelling (FDM) machine. In our previous study, a NFRPC material was made from recycled polystyrene and corn husk fibre via compression moulding method [5]. Then, the similar composite material also made into FDM filament and used for FDM printing. The 3D printed components with this composite material exhibited a good mechanical strength and high stiffness as compared to commercial plastic filament [6]. The study related to NFRPC

\footnotetext{
* Corresponding author: seongchun.koay@taylors.edu.my
} 
filament is very rare, thus this study was carried out to explore its potential for lattice structure application.

Additive manufacturing is a current famous manufacturing process used to fabricate a $3 \mathrm{D}$ component by applying a thin layer of material over another [7-8]. This processing method widely used to produce component with cellular structure. Cellular pattern also called as lattice structure. The lattice structure exhibits an advantage in additive manufacturing process, this is because it enhanced the component's stiffness, weight to strength ratios, reduce the printing time for 3D printing and generate minimum of waste due to less support. The stochastic and non-stochastic lattice structures are widely used in cellular patter design. Stochastic lattice structure usually is randomly designed structure and non-stochastic lattice structure are patterned design structure with shape of prism, such as triangle prism, hexagonal prism and square prism [9-10]. There are many studies related to study of lattice structure via FDM process usually used materials, such as acrylic butadiene styrene (ABS) [10]. However, the study of lattice structure using NFRPC material is not found in any open-source literatures. Thus, this research was studying the lattice structure made from corn husk fibre reinforced recycled polystyrene composite.

Lattice structure usually designed with the aim of Computer Aided Design (CAD) software, such as $\mathrm{Creo}^{\circledR}$. The main challenge in designing the lattice structure is the mechanical variation influenced by the shapes and sizes of the struts building the lattice due to the processability of $3 \mathrm{D}$ printing process. Some researchers have focused on the assessment of mechanical properties of lattice structures using FEA method [9]. Niu et al. [10] demonstrated a FEA studied on different shape and size of prism on lattice structure. They able to find the best shape and size of prism for a lattice structure with maximum stiffness-to-mass ratio. Thus, this study also uses FEA simulation to study the mechanical properties of lattice structure for NFRPC material.

This research focused on triangular prism and hexagonal prism for the lattice structure in this work. This research used FEA to assess the mechanical properties of the lattice structure made from corn husk fibre reinforced recycled polystyrene composite material.

\section{Methodology}

The 3D model of the lattice structures was created using $\mathrm{Creo}^{\circledR}$ software. The specimen was a rectangular in shape with dimension of $25 \mathrm{~mm} \times 10 \mathrm{~mm} \times 5 \mathrm{~mm}$ and it composed of either hexagonal prism or triangular prism as shown in Figure 1. In this research, ANSYS R19 version software was used for the simulation. The models were used tetrahedron element and the mesh quality were controlled more than 0.8. In order to study the mechanical behaviour of the model, the bottom face of the model was be assigned as fixed support and force applied on opposite face which acting upwards on the surface of the model as displayed in Figure 2. The applied force was 100N.
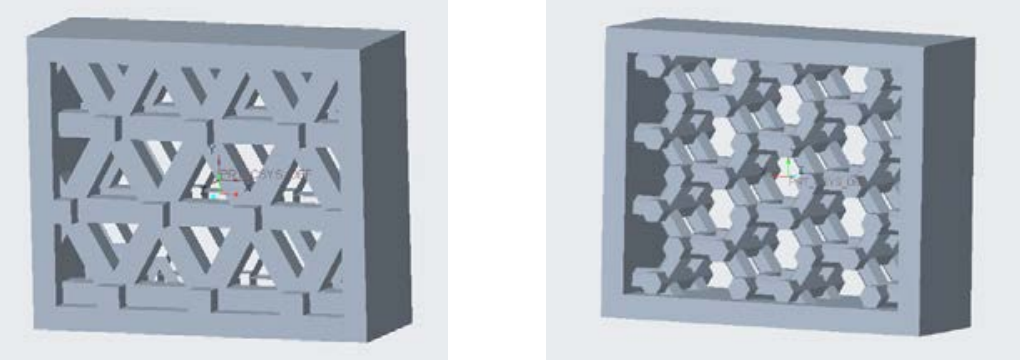

Fig. 1. The model of lattice cell with triangular prism (left) and hexagonal prism (right) 


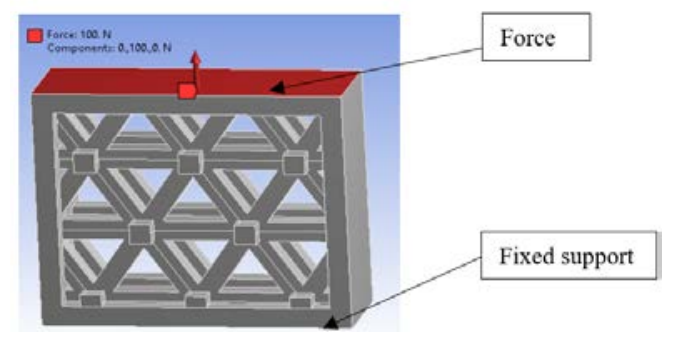

Fig. 2. Model assigned fixed support and force.

The composite material used for this study was corn husk fibre reinforced recycled polystyrene. This composite material containing $7.5 \mathrm{wt} \%$ of corn husk fibre with tensile strength of $24 \mathrm{MPa}$ and elastic modulus of $2.4 \mathrm{GPa}$. The poison ratio of the material is 0.35 . The mechanical properties of this composite material were obtained from study reported by Ariel et al. [6]. The simulation on the lattice structure was focused on 3 different conditions.

First condition, the models with triangular prism and hexagonal prism were created according to parameter listed in Table 1. Both models have similar surface area. Figure 3 shows the measure of parameters for hexagonal and triangular prisms. Figure 4 illustrates the lattice parameter for triangular prism and hexagonal prism. The thickness, $t$ was thickness of the strut, $L$ was length of the prism and $h$ was height of the prism. The surface area of both type of prisms was calculate using eq. (1) and eq. (2).

$$
\text { Area of hexagon, } \mathrm{A}_{\mathrm{H}}=\frac{6}{2} \times \mathrm{hL}=\frac{3 \sqrt{3} \mathrm{~L}^{2}}{2}
$$

$$
\text { Area of triangle, } \mathrm{A}_{\mathrm{T}}=\frac{\sqrt{3}}{4} \mathrm{~L}^{2}=\frac{1}{2} \mathrm{Ly}
$$

For second condition, the parameter $L$ and $h$ were fixed at $2 \mathrm{~mm}$. Then, thickness of the prism was different at $2 \mathrm{~mm}, 2.2 \mathrm{~mm}$. and $2.4 \mathrm{~mm}$. For third condition, the models with hexagonal prism and triangular prism were created using parameter, $L=8 \mathrm{~mm}, h=5 \mathrm{~mm}$ and $t=2 \mathrm{~mm}$.

Table 1. Lattice parameter for first condition.

\begin{tabular}{|l|c|c|c|c|c|c|}
\hline $\begin{array}{c}\text { Type of } \\
\text { prism }\end{array}$ & $\boldsymbol{L}, \mathbf{m m}$ & $\boldsymbol{H}, \mathbf{m m}$ & $\boldsymbol{t}, \mathbf{m m}$ & $\boldsymbol{x}, \mathbf{m m}$ & $\boldsymbol{y}, \mathbf{m m}$ & $\begin{array}{c}\text { Surface Area, } \\
\mathbf{m m}^{2}\end{array}$ \\
\hline Hexagonal & 4 & 5 & 2 & 8 & 6.9282 & 41.57 \\
\hline Triangular & 9.798 & 5 & 2 & 9.798 & 8.49 & 41.57 \\
\hline
\end{tabular}

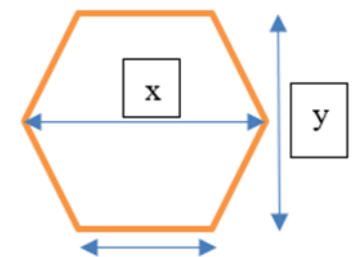

$\mathrm{L}$

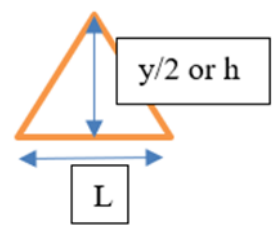

Fig. 3. The relation between parameter of hexagonal prism (left) and triangular prism (right). 

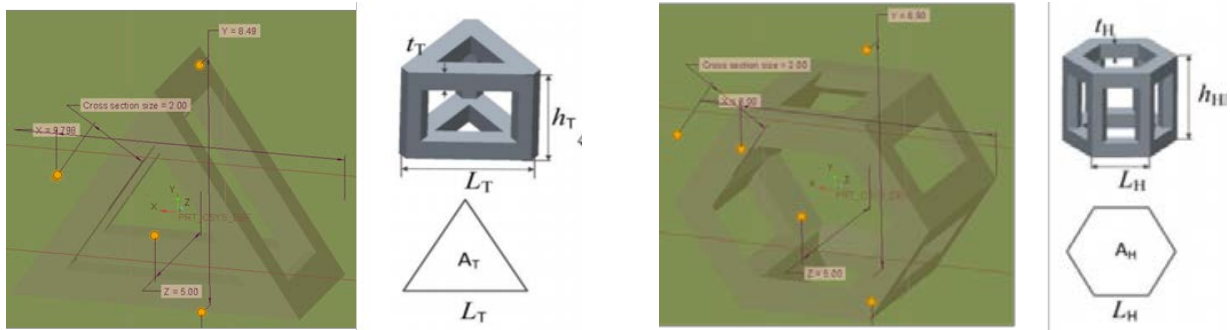

Fig. 4. The parameter of triangular prism (left) and hexagonal prism (right)

\section{Result and Discussion}

Figures 5 and 6 display the normal stress and total deformation of lattice structure with hexagonal prism and triangular prism with similar surface area. At similar surface area, the lattice structure with triangular prism experienced less maximum normal stress compared to lattice structure with normal stress. This indicated that the lattice structure with triangular prism was much stronger than the lattice structure with lattice structure with hexagonal prism. Furthermore, the lattice structure with triangular prism also exhibited $50 \%$ less maximum total deformation than lattice structure with hexagonal prism. This means the lattice structure with triangular prism exhibited higher stiffness compared to lattice structure with hexagonal prism.

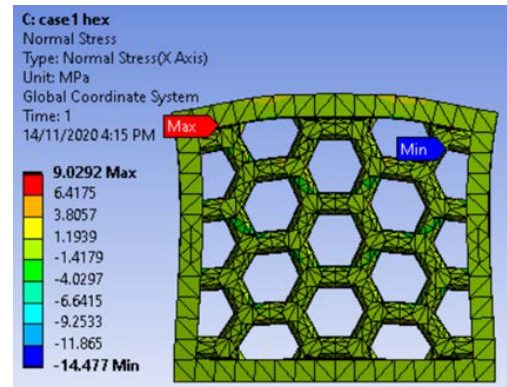

Max Normal Stress: $9.0292 \mathrm{MPa}$

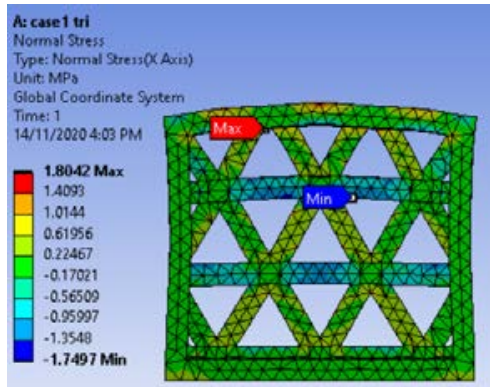

Max Normal Stress: $1.8042 \mathrm{MPa}$

Fig. 5. Normal stress on lattice cell with hexagonal prism (left) and triangular prism (right) at similar surface area

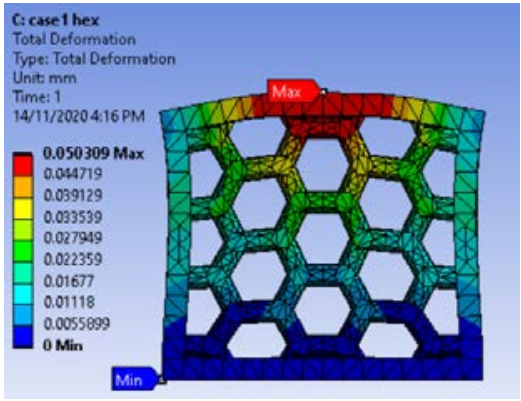

Max total deformation : $0.05 \mathrm{~mm}$

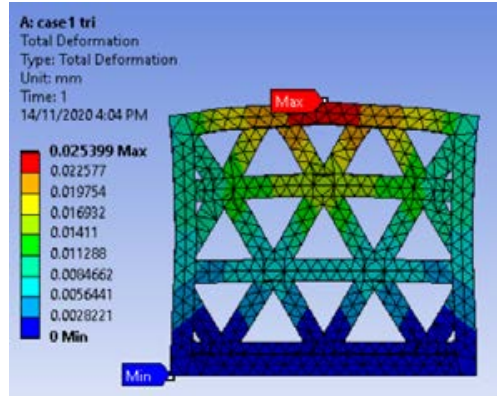

Max total deformation : $0.025 \mathrm{~mm}$

Fig. 6. Total deformation on lattice cell with hexagonal prism (left) and triangular prism (right) at similar surface area

Figure 7 illustrates the max normal stress and max total deformation of lattice structure with hexagonal prism and triangular prism with different thickness, $t$. The 
increases of thickness of the struts significantly reduced the maximum normal stress and maximum total deformation of lattice structure. This also mean the lattice structure need more material to build. The thicker struts significantly enhanced the overall strength and stiffness of the lattice structure. In additional, the maximum normal stress and maximum total deformation of lattice structure with triangular have decreased approximately $62 \%$ and $42 \%$, respectively, when the strut thickness increased from $2 \mathrm{~mm}$ to $2.4 \mathrm{~mm}$. In opposite, the lattice structure with triangular prism exhibited about $33 \%$ and $32 \%$, respectively, deduction in maximum normal stress and maximum total deformation after thickness increased. As the struct thickness increases, the lattice structure with triangular prism experience lesser deduction in maximum normal stress and maximum total deformation compared to lattice structure with hexagonal prism. This indicated that the lattice structure with triangular prism can be built with lesser material and the structure still stronger and stiffer than lattice structure with hexagonal prism.
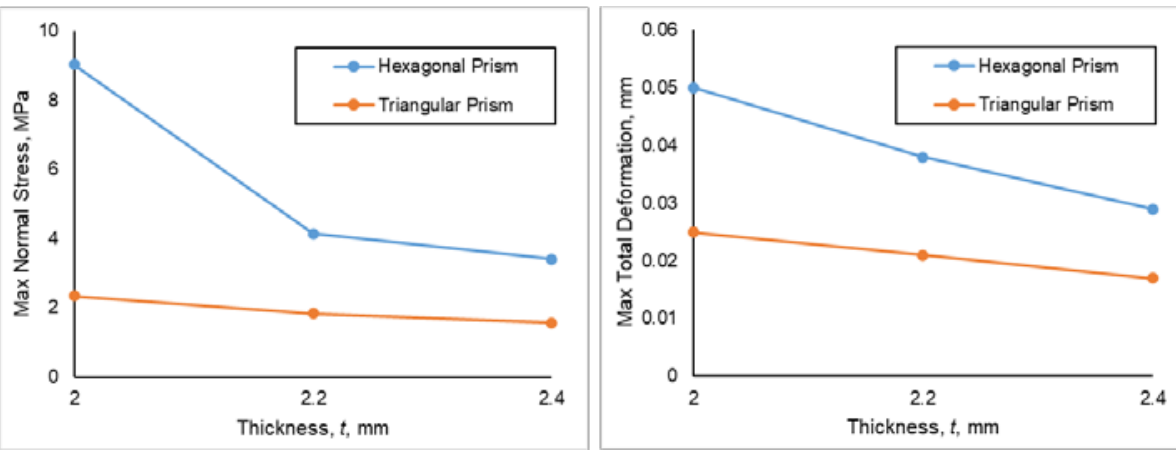

Fig. 7. Max normal stress (left) and max total deformation (right) of lattice cell with hexagonal prism and triangular prism (right) with different thickness $(t)$.

The visual results of normal stress and total deformation of lattice structure with hexagonal prism and triangular prism with similar lattice parameter are showed in Figures 8 and 9 . When both lattice structure having similar lattice parameter, the lattice structure with triangular prism displayed lower maximum normal stress and maximum total deformation than lattice structure with hexagonal prism. This indicates the lattice structure with triangular prism has approximately $50 \%$ and $62 \%$, respectively, higher strength and stiffness than the lattice structure with hexagonal prism. Niu et al. [10] also reported that the lattice structure with triangular prism exhibited higher strength and elastic modulus compared to lattice structure with hexagonal prism.

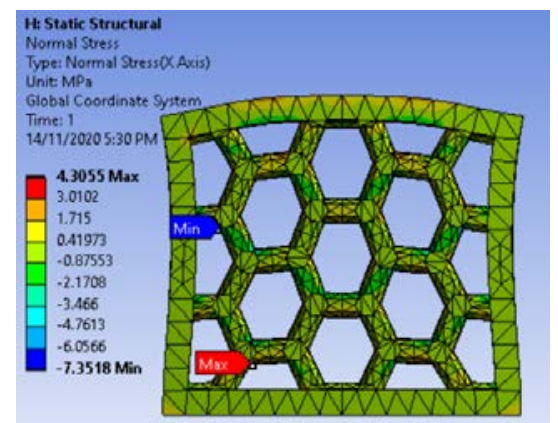

Max Normal Stress: $4.3055 \mathrm{MPa}$

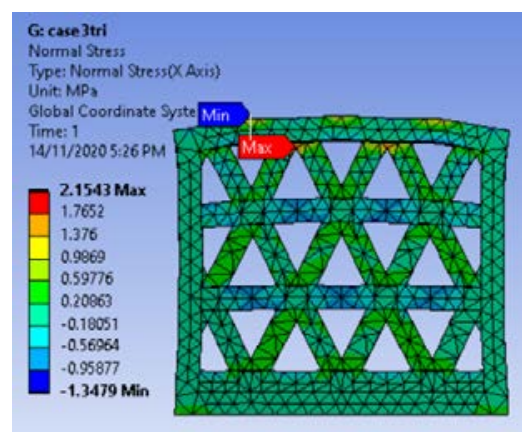

Max Normal Stress: $2.143 \mathrm{MPa}$

Fig. 8. Normal stress on lattice cell with hexagonal prism (left) and triangular prism (right) at similar lattice parameter 


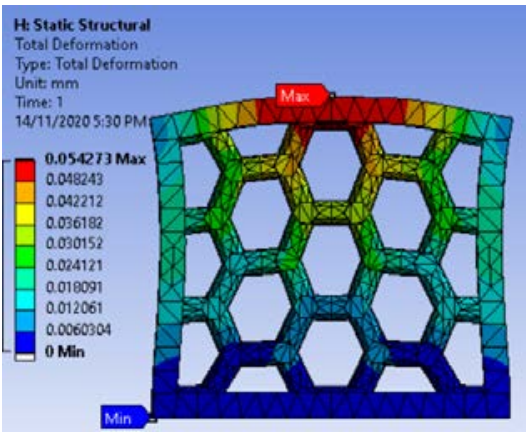

Max total deformation : $0.054273 \mathrm{~mm}$

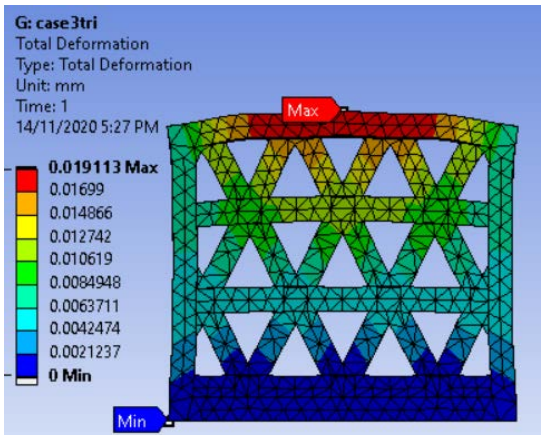

Max total deformation : $0.019113 \mathrm{~mm}$

Fig. 9. Total deformation on lattice cell with hexagonal prism (left) and triangular prism (right) at similar lattice parameter

\section{Conclusion}

The FEA simulation results indicated that the lattice structure with triangular prism exhibited better strength and stiffness than lattice structure with hexagonal prism. The lattice structure with triangular prism also shows lesser reduction in maximum normal stress and maximum total deformation compared to lattice structure with hexagonal prism. Therefore, lattice structure with triangular prism can be fabricated via FDM process with lesser corn husk fibre reinforced recycled polystyrene composite material, but the strength and stiffness of the structure can be better than lattice structure with hexagonal prism.

\section{References}

1. K.S. Chun, T. Maimunah, C.M. Yeng, T.K. Yeow, O.T. Kiat, J. Phy. Sci. 31, 17 (2020).

2. M.C. Lee, K.S. Chun, M.Y. Chan, H.L. Choo, M.M. Pang, K.Y. Tshai, J. Thermoplast. Compos. Mater. 33, 1518 (2020).

3. S.L. Ling, S.C. Koay, M.Y. Chan, K.Y. Tshai, T.R. Chantara, M.M. Pang, Polym. Eng. Sci. 60, 202 (2020).

4. S.C. Koay, M.Y. Chan, M.M. Pang, K.Y. Tshai, Adv. Polym. Techn. 37, 2246 (2018)

5. K.S. Chun, N.M.Y. Fahamy, C.M. Yeng, K.Y. Tshai, M.M Pang, H.L Choo, J. Eng. Sci. Techn. 13, 3445 (2018).

6. L.J.J. Ariel, S.C. Koay, H.L. Choo, T.K. Ong, in $13^{\text {th }}$ Int. Eng. Res. Conf. (2019).

7. T.Y. Ng, S.C. Koay, M.Y. Chan, H.L. Choo, T.K. Ong, AIP Conf. Proc. 2233, 020022 (2020).

8. T.K. Ong, H.L. Ong, W.J. Choo, S.C. Koay, M.M. Pang, Adv. Manuf. Eng. 725 (2020).

9. J. Niu, H. L. Choo, W. Sun, S.H. Mok, Int. J. Mech. Mater. Des. 14, 443 (2018).

10. J. Niu, H.L. Choo, W. Sun, Proc. IMechE Part L: J. Mater. Des Appl. 231, 1 (2016). 